FIT HE - 03-05

\title{
Effective action and brane running
}

\author{
Iver Brevik ${ }^{1}$ \\ Department of Energy and Process Engineering, Norwegian University of Science and \\ Technology, N-7491 Trondheim, Norway \\ Kazuo Ghoroku \\ ${ }^{2}$ Fukuoka Institute of Technology, Wajiro, Higashi-ku \\ Fukuoka 811-0295, Japan \\ Masanobu Yahiro ${ }^{3}$ \\ ${ }^{4}$ Department of Physics and Earth Sciences, University of the Ryukyus, \\ Nishihara-chou, Okinawa 903-0213, Japan
}

\begin{abstract}
We address the renormalized effective action for a Randall-Sundrum brane running in $5 \mathrm{~d}$ bulk space. The running behavior of the brane action is obtained by shifting the brane-position without changing the background and the fluctuations. After an appropriate renormalization, we obtain an effective, low energy braneworld action, in which the effective 4d Planck mass is independent of the running-position. We address some implications of this effective action.
\end{abstract}

\footnotetext{
${ }^{1}$ iver.h.brevik@mtf.ntnu.no

${ }^{2}$ gouroku@dontaku.fit.ac.jp

3 yahiro@sci.u-ryukyu.ac.jp
} 


\section{Introduction}

It is quite probable that our $4 \mathrm{~d}$ world is formed according to a ten-dimensional superstring theory. In particular, an interesting geometry $A d S_{5} \times S^{5}$ is obtained near the horizon of the stacked D3-branes in type IIB string theory. And the string theory on this background may describe a four-dimensional conformal field theory (CFT), $\mathcal{N}=4$ SUSY Yang-Mills theory, which lives on the boundary [1, 2, 3, 4,

On the other hand, a thin three-brane (Randall-Sundrum brane) embedded in $A d S_{5}$ space [5, 6] can be regarded as the boundary pushed out to AdS bulk. Since the coordinate transverse to the brane is considered as the energy scale of CFT on the boundary, the position of the brane represents the ultraviolet cut-off scale of the CFT.

Usually, the brane action is expressed in terms of a tension only, when solving the equation of motion for the braneworld. It has recently been pointed out that many higher derivative terms appear in the brane action when we make a change of the brane position without changing the solutions for the background and the KaluzaKlein (KK) modes [7, 8, 9, 10]. This procedure is called brane running and is considered as an approximate form of obtaining renormalization group flow. This implies that the parameters of the brane-action at a different position could be related through this renormalization group flow. Inspired by this idea, we examine the running behavior of the brane action and derive the effective braneworld action with an appropriate renormalization.

We restrict ourselves to the case of pure gravity in the $5 \mathrm{~d}$ bulk. It is well known that the zero mode is trapped on the brane and the usual $4 \mathrm{~d}$ Newton's law can be observed on the brane. Non-trapped, massive KK modes are also observed through a correction to Newton's law, and this is also understood from the $4 \mathrm{~d}$ field theory based on the AdS/CFT correspondence [11, 12]. We address the effects of KK modes through the running brane-action, which is obtained as a solution of the flow equations. But ultra-violet divergences are generally included in the brane-action. This is generally expected from the viewpoint of higher dimensional field theory, which would correspond to CFT on the brane, since loop-corrections are in general included in the brane action [13, 14, 15]. Then the effective action is obtained after renormalizing the running brane-action in a consistent way via the AdS/CFT correspondence. When we set the brane near the boundary of $\mathrm{AdS}_{5}$, the higher derivative terms are neglected and we arrive at an low energy, effective braneworld action in which the effective 4d Planck mass is independent of the flow-position. In other words, the original Randall-Sundrum formulation, in which the brane action is written in terms of tension only, is available at any flowing position as far as low energy theory is considered.

In Section 2, the model used here is set, and the flow equation for the tension is derived and solved in Section 3. In Section 4, the flow equations for the brane action are derived and solved. The effective brane action is derived by an appropriate renormalization condition, and its meaning is discussed. In Section 5, the case of two parallel branes is discussed and the problem of their distance is commented upon. 
Concluding remarks are given in the final section.

\section{Setting of the brane-world}

We start from the five-dimensional gravitational action. It is given in the Einstein frame $\mathrm{as}^{4}$

$$
S_{5}=\frac{1}{2 \kappa^{2}}\left\{\int d^{5} X \sqrt{-G}(R-2 \Lambda)+2 \int d^{4} x \sqrt{-g} K\right\},
$$

where $K$ is the extrinsic curvature on the boundary. The other ingredient is the brane action,

$$
S_{\mathrm{b}}=-\tau \int d^{4} x \sqrt{-g},
$$

which is added to $S_{5}$, and the Einstein equation is obtained as

$$
R_{M N}-\frac{1}{2} g_{M N} R=\kappa^{2} T_{M N}
$$

where $\kappa^{2} T_{M N}=-\left(\Lambda+b \delta(y) \kappa^{2} \tau \delta_{\mu}^{M} \delta_{\nu}^{N}\right) g_{M N}$ and $b=\sqrt{-g} / \sqrt{-G}$. Here we solve the Einstein equation (3) with the following metric,

$$
d s^{2}=A^{2}(y)\left\{-d t^{2}+a_{0}^{2}(t) \gamma_{i j}\left(x^{i}\right) d x^{i} d x^{j}\right\}+d y^{2},
$$

where the coordinates parallel to the brane are denoted by $x^{\mu}=\left(t, x^{i}\right), y$ being the coordinate transverse to the brane. The position of the brane is taken at $y=0$. We restrict our interest here to the case of a Friedmann-Robertson-Walker type (FRW) universe. Then, the three-dimensional metric $\gamma_{i j}$ is described in Cartesian coordinates as

$$
\gamma_{i j}=\left(1+k \delta_{m n} x^{m} x^{n} / 4\right)^{-2} \delta_{i j}
$$

where the parameter values $k=0,1,-1$ correspond to a flat, closed, or open universe respectively.

When considering the metric (44), we obtain the following reduced equations [16]:

$$
\left(\frac{\dot{a_{0}}}{a_{0}}\right)^{2}+\frac{k}{a_{0}^{2}}=A^{\prime 2}+\frac{\Lambda}{6} A^{2}=D
$$

where $D$ is a constant being independent of $t$ and $y$. In view of the boundary condition at the brane position,

$$
A^{\prime}(0)=-\frac{\kappa^{2} \tau}{6} A(0)
$$

\footnotetext{
${ }^{4}$ Here we take the following definition, $R_{\nu \lambda \sigma}^{\mu}=\partial_{\lambda} \Gamma_{\nu \sigma}^{\mu}-\cdots, R_{\nu \sigma}=R_{\nu \mu \sigma}^{\mu}$ and $\eta_{A B}=\operatorname{diag}(-1,1,1,1,1)$. Five dimensional suffices are denoted by capital Latin and four dimensional ones by Greek letters.
} 
one gets

$$
D \equiv \lambda=\kappa^{4} \tau^{2} / 36+\Lambda / 6
$$

if $A(0)=1$. Here we use the following notation, ${ }^{\prime}=d / d t$ and ${ }^{\prime}=d / d y$. The normalization condition $A(0)=1$ does not affect the generality of our discussion.

Here $\lambda$ denotes the cosmological constant on the brane as seen from the first equation of (6),

$$
\left(\frac{\dot{a_{0}}}{a_{0}}\right)^{2}+\frac{k}{a_{0}^{2}}=\lambda
$$

While the tension of the brane is expressed by this $\lambda$ according to (8),

$$
\tau=\frac{6 \mu}{\kappa^{2}} \sqrt{1+\frac{\lambda}{\mu^{2}}}, \quad \mu=\sqrt{-\frac{\Lambda}{6}} .
$$

This result is obtained for a fixed brane-position at $y=0$, but the position is arbitrary in the above discussion. In the context of AdS/CFT correspondence, the coordinate $y$ has the meaning of an energy-scale in the field theory on the brane. We can obtain some information about renormalization group flow of the field theory when the brane at different positions of $y$ are related by an appropriate principle. In the following, we study this problem by using the boundary conditions of the field equations and by extending the brane action such that it can change with $y$.

\section{Flow of $\tau$}

In the previous section, $\tau$ in (10) is obtained in terms of (21) and (7) for the braneposition $y_{0}=0$. Here we consider a shift the position from $y_{0}$ to $y_{1}=y_{0}+\epsilon$ without changing the bulk configuration $A(y)$. Then we obtain the following boundary condition, which can be written in the same form as (77),

$$
A^{\prime}\left(y_{1}\right)=-\frac{\kappa^{2} \tau\left(y_{1}\right)}{6} A\left(y_{1}\right)
$$

Here we have replaced $\tau$ by $\tau(y)$, a function of $y$. In other words, this shift describes the change of $\tau(y)$ with $y$ in accordance with the change of $A(y)$ with $y$. This means that the parameter of the brane action must run with $y$ so as to satisfy (11).

Then for the shift of $y_{0}$ keeping the bulk configuration unchanged, $\tau(y)$ is obtained for any value of $y$ as

$$
\tau(y)=-\frac{6}{\kappa^{2}} \frac{A^{\prime}(y)}{A(y)} .
$$

This result can be also obtained by solving a differential equation that is derived by expanding (11) in a series of $\epsilon$ when we take $y_{1}=y_{0}+\epsilon$. It is given as

$$
\tau^{\prime}=\frac{\Lambda}{\kappa^{2}}+\frac{\kappa^{2}}{6} \tau^{2}
$$


In getting this equation, we used the Einstein equations for $A$. It is easy to show the equivalence of the solution of this equation and the one given by (12). This is because $A$ obeys the following linear equation,

$$
\frac{A^{\prime \prime}}{A}+\frac{\Lambda}{6}=-\frac{\kappa^{2} \tau}{3} \delta(y)
$$

whereby $A^{\prime \prime}$ can be rewritten in terms of $A$.

We will consider some explicit examples of flows mentioned above, in terms of typical brane-world solutions.

\subsection{Solutions for $\lambda=0$}

When $\lambda=0$, the solution is given by

$$
d s^{2}=e^{-2 \mu|y|}\left(-d t^{2}+\delta_{i j} d x^{i} d x^{j}\right)+d y^{2} .
$$

In this case $A=e^{-\mu|y|}$, and we obtain from (12)

$$
\tau(y)=\frac{6 \mu}{\kappa^{2}}
$$

This corresponds to a fixed point in the sense of flow of $\tau$ as we should expect, i.e.,

$$
\tau^{\prime}=0
$$

This is also assured from (13) by adopting the "initial" value of $\tau$ as $\tau(0)=\frac{6 \mu}{\kappa^{2}}$.

However, this does not mean that there is no $y$-dependent terms in the brane action. As shown in (23) in the next subsection, the general form of the brane action could include many terms, but at present we see only the first term $\tau_{0}$ appearing in (23). Actually, we can see other $y$-dependent terms from the solution of the "RG" equation derived from the gravitational fluctuations. We will show this in the next section.

\subsection{Solutions for $\lambda>0$ and $\Lambda<0$}

For $\lambda=0$, there was no special position other than the position of the brane, $y_{0}$. But for $\lambda>0$ a horizon $y_{H}$, where $A\left(y_{H}\right)=0$, appears. We consider the region of $y_{H}>y_{0}$ hereafter.

When $\lambda>0$ and $\Lambda$ is negative, one has

$$
\begin{gathered}
A(y)=\frac{\sqrt{\lambda}}{\mu} \sinh \left[\mu\left(y_{H}-|y|\right)\right], \\
\sinh \left(\mu y_{H}\right)=\mu / \sqrt{\lambda} .
\end{gathered}
$$


Here $y_{H}$ represents the horizon in the bulk $\mathrm{AdS}_{5}$, and (19) is a condition for $A(0)=1$. However, we notice that $A(0)$ might be arbitrary and $A(0)=1$ is at present chosen for simplicity. This point will be discussed below. We get

$$
\tau(y)=\frac{6 \mu}{\kappa^{2}} \operatorname{coth}\left(\mu\left[y_{H}-y\right]\right)
$$

which gives interesting information. In view of (19) we obtain the following expansion of $\tau$ with respect to $\lambda$,

$$
\begin{aligned}
\tau(y)= & \frac{6 \mu}{\kappa^{2}} \sqrt{1+\frac{\lambda}{\mu^{2} A^{2}}} \\
& =\frac{6 \mu}{\kappa^{2}}+\frac{3}{\kappa^{2} \mu} \frac{\lambda}{A^{2}}+O\left(\lambda^{2}\right) .
\end{aligned}
$$

The first line of the above equation is written as

$$
\left(\frac{\kappa^{2} \tau}{6}\right)^{2}=\mu^{2}+\frac{\lambda}{A^{2}}
$$

which represents the extended form of the parameter relation obtained at $y=0$, $\left(\frac{\kappa^{2} \tau}{6}\right)^{2}=\mu^{2}+\lambda$, to that holding at arbitrary $y$.

As for the expansion in the second line, we suggest that the origin of the $\lambda$-dependent terms is as follows. Consider the following form of brane action including curvature terms,

$$
\tilde{S}_{\mathrm{b}}=-\int d^{4} x \sqrt{-g}\left(\tau_{0}+\tau_{1} R+\tau_{2}\left(R_{\mu \nu} R^{\mu \nu}-\frac{1}{3} R^{2}\right)+\tilde{\tau}_{2} R^{2}+\cdots\right) .
$$

Then we find the following form of $\tau$,

$$
\tau=\tau_{0}+\tau_{1} \frac{6 \lambda}{A^{2}}+O\left(\lambda^{3}\right),
$$

for the de Sitter brane. For the case of Minkowski brane, $\tau=\tau_{0}$, as stated above. In the case of $\lambda>0, \tau$ should be constructed by many curvature terms in general, if they were prepared in the brane action. If $\tau_{i}(y), i \geq 1$ does not include $\lambda$, we find

$$
\tau_{0}=\frac{6 \mu}{\kappa^{2}}, \quad \tau_{1}=\frac{1}{2 \kappa^{2} \mu}, \quad \cdots
$$

We should notice that the first term is consistent with the one given in the previous sub-section. However, it turns out that the second term depends on $y$, as is shown in the next section. Then $\tau_{i}$ themselves must depend on $\lambda$, and we will need an infinite series of curvatures in the brane action to allow for the shift of brane position without changing the background configuration. This is seen more explicitly in the next section when the same condition is imposed on equations of the field-fluctuations.

For the cases of other brane solutions, we give a brief comment in the final section. 


\section{Flow of $\tau_{i}, i \geq 1$, and effective brane action}

Consider the perturbed metric $h_{i j}$ in the form

$$
d s^{2}=A^{2}(y)\left(-d t^{2}+a_{0}^{2}(t)\left[\gamma_{i j}\left(x^{i}\right)+h_{i j}\left(y, x^{\mu}\right)\right] d x^{i} d x^{j}\right)+d y^{2} .
$$

We are interested in the traceless transverse component, which represents the graviton on the brane, of the perturbation. It is projected out by the conditions, $h_{i}^{i}=0$ and $\nabla_{i} h^{i j}=0$, where $\nabla_{i}$ denotes the covariant derivative with respect to the three-metric $\gamma_{i j}$ which is used to raise and lower the three-indices $i, j$. The transverse and traceless part is denoted by $h$ hereafter for simplicity.

We first consider the simple case where $\Lambda<0$ and $\gamma_{i j}\left(x^{i}\right)=\delta_{i j}$. Then the transverse and traceless part $h$ is projected out by $\partial_{i} h^{i j}=0$ and $h_{i}^{i}=0$, where $\delta_{i j}$ is used to raise and lower the indices $i j$. One arrives at the following linearized equation of $h$ in terms of the five (four) dimensional covariant derivative $\nabla_{5}^{2}=\nabla_{M} \nabla^{M}\left(\nabla_{4}^{2}=\nabla_{\mu} \nabla^{\mu}\right)$ :

$$
\frac{1}{2 \kappa^{2}} \nabla_{5}^{2} h=\left(\tau_{1} \frac{\nabla_{4}^{2}}{A(0)^{2}}-\tau_{2} \frac{\nabla_{4}^{4}}{A(0)^{4}}+\cdots\right) h \delta(y) .
$$

We notice that $\tau_{0}$ is not included on the right hand side since it disappears due to the classical Einstein equation. And the right hand side is expressed exactly for $\lambda=0$. When $\lambda$ is finite, $\tau_{i}$ should be replaced by $\bar{\tau}_{i}$ which include the terms coming from higher curvature terms like $R^{i+n}(n \geq 1)$. In this sense, we can not see the pure $\tau_{i}$ for the case of $\lambda>0$. For a while, we denote $\tau_{i}$ by the same notation for $\lambda=0$ and $\lambda>0$.

Eq. (27) is rewritten as follows by the following factorization of $h$,

$$
h=\int d m \phi_{m}\left(t, x^{i}\right) \Phi(m, y),
$$

where the mass $m$ is defined by

$$
\ddot{\phi}_{m}+3 \frac{\dot{a}_{0}}{a_{0}} \dot{\phi}_{m}+\frac{-\partial_{i}^{2}}{a_{0}^{2}} \phi_{m}=-m^{2} \phi_{m}
$$

then the equation for $\Phi(m, y)$ is obtained as

$$
\Phi^{\prime \prime}+4 \frac{A^{\prime}}{A} \Phi^{\prime}+\frac{m^{2}}{A^{2}} \Phi=2 \kappa^{2}\left(\tau_{1} \frac{m^{2}}{A(0)^{2}}-\tau_{2} \frac{m^{4}}{A(0)^{4}}+\cdots\right) \Phi(0) \delta(y) .
$$

In solving (30), we need the following boundary condition,

$$
\frac{\Phi^{\prime}(0)}{\Phi(0)}=\kappa^{2}\left(\tau_{1} \frac{m^{2}}{A^{2}(0)}-\tau_{2} \frac{m^{4}}{A^{4}(0)}+\cdots\right) .
$$

Then this boundary condition is different from the one used before [16], where we used $\tau_{i}=0, i \geq 1$. The new boundary condition affects only the KK modes, but it does not 
give any effect on the zero mode. Then the results for the localization of graviton, the zero mode, are not changed even if we consider the above new boundary conditions.

By shifting the position of brane, we obtain flow equations for $\tau_{i}$ as above by defining $\tau_{T}=-\tau_{1} \frac{m^{2}}{A^{2}}+\tau_{2} \frac{m^{4}}{A^{4}} \cdots$,

$$
\tau_{T}^{\prime}=-4 \frac{A^{\prime}}{A} \tau_{T}+\kappa^{2} \tau_{T}^{2}+\frac{m^{2}}{\kappa^{2} A^{2}}
$$

¿From this, flow equations for $\tau_{i}$ are obtained as,

$$
\begin{aligned}
& \tau_{1}^{\prime}=-2 \frac{A^{\prime}}{A} \tau_{1}-\frac{1}{\kappa^{2}}, \quad \tau_{2}^{\prime}=\kappa^{2} \tau_{1}^{2}, \\
& \tau_{3}^{\prime}=-2 \kappa^{2} \tau_{1} \tau_{2}+2 \frac{A^{\prime}}{A} \tau_{3}, \quad \cdots
\end{aligned}
$$

\subsection{The case $\lambda=0$}

In this case, $\tau_{1}$ is obtained as,

$$
\tau_{1}=\frac{1}{2 \mu \kappa^{2}}+c_{1} e^{2 \mu y}
$$

with an integration constant $c_{1}$. For $\mathrm{AdS}_{5}$ background, $\tau$ is independent of $y$ as seen in the previous section. So one idea to determine $c_{1}$ is to demand that $\tau_{1}$ is also $y$ independent. This is realized by choosing $c_{1}=0$. However, $\tau_{2}$ should be running for the above setting of $\tau_{1}$ since the second equation of (33) is written as

$$
\tau_{2}^{\prime}=\frac{1}{4 \mu^{2} \kappa^{2}}
$$

and this is solved as

$$
\tau_{2}=\frac{1}{4 \mu^{2} \kappa^{2}} y+c_{2}
$$

where $c_{2}$ is a constant. This term is interpreted as the anomaly produced by oneloop CFT, from the viewpoint of $\mathrm{AdS} / \mathrm{CFT}$ correspondence. In this sense, there is no $y$-independent solution of $\tau_{i}, i \geq 1$. In other words, there is no fixed point in the parameter space.

Further, we obtain $\tau_{3}$ as

$$
\tau_{3}=c_{3} e^{-2 \mu y}-\frac{1}{8 \mu^{4} \kappa^{2}} y+\tilde{c}_{2}
$$

where $\tilde{c}_{2}=-c_{2} /\left(2 \mu^{2}\right)+1 /\left(16 \mu \kappa^{2}\right)$. In the context of the renormalization group, this corresponds to an irrelevant term. But it is running, as it has received a quantum 
correction from CFT similarly to $\tau_{2}$. We can see the running of other irrelevant terms as well as $\tau_{3}$. Then the flow equations given here would be valid as renormalization group equations in the region where the irrelevant terms vanish or are suppressed.

The first term of $\tau_{3}$ becomes small when the brane position $y_{0}$, which is set as $y_{0}=0$ in equation (38), is taken near the boundary, $y_{0}=-\infty$, since it is written as $c_{3} e^{-2 \mu\left(y-y_{0}\right)}$ in this case ${ }^{5}$. So we can neglect this term near the boundary. The remaining terms could be suppressed under the condition that the above flow equations are valid for small $\mathrm{m}^{2} / \mu^{2}$ or for small mass of the KK modes compared to $\mu$. Then we can neglect the irrelevant terms for $m^{2} / \mu^{2}<<1$. Even if $\tau_{i}, i \geq 3$ were remaining finite, these irrelevant terms would vanish in the action due to the warp factor $A^{-2 i+4}$ in the limit of $y_{0} \rightarrow-\infty$. In this sense, the effective action is well defined near the boundary in terms of the relevant terms. Hereafter, we consider the braneworld including only low frequency KK modes of $m^{2} / \mu^{2}<<1$ to remove the irrelevant terms in the effective braneworld action.

Then we estimate the effective brane action $S_{b}^{\text {eff }}$ from the viewpoint of AdS/CFT correspondence neglecting the irrelevant terms. It can be written as [17, 18, 19, 20]

$$
\begin{gathered}
S_{b}^{\mathrm{eff}}=\frac{1}{2} \tilde{S}_{b}+\ln Z_{5}(g)=\frac{1}{2} \tilde{S}_{b}+S_{\mathrm{CT}}+S_{\mathrm{CFT}} \\
Z_{5}(g)=\int_{\left.G\right|_{y=0}=g} D G D \psi e^{i S_{5}},
\end{gathered}
$$

where $\tilde{S}_{b}$ and $S_{5}$ are given in the previous section. The fields other than the metric contained in $S_{5}$ are denoted by $\psi$. In the case of $\operatorname{AdS}_{5}$, we can set $\tau_{0}=\tau$, then $\tilde{S}_{b}$ can be given by solving the above flow equation for other $\tau_{i}$. The term $\ln Z_{5}(g)$ is replaced by $S_{\mathrm{CT}}$ (the counter term) and $S_{\mathrm{CFT}}$ (cut-off CFT), which interacts with the gravity in $\tilde{S}_{b}$ and provides the quantum correction to $\tau_{2}$. The term $S_{\mathrm{CT}}$ is given as,

$$
S_{\mathrm{CT}}=\int d^{4} x \sqrt{-g}\left(\frac{3 \mu}{\kappa^{2}}+\frac{1}{4 \mu \kappa^{2}} R+\frac{\ln (\mu z)}{8 \mu^{3} \kappa^{2}}\left(R_{\mu \nu} R^{\mu \nu}-\frac{1}{3} R^{2}\right)\right),
$$

where $\mu y=\ln (\mu z)$.

As for $\tilde{S}_{b}$, we consider the boundary condition of the usual brane model, $\tau_{i}(0)=$ $0, i \geq 1$. Then $c_{1}=-1 /\left(2 \mu \kappa^{2}\right)$. By demanding also $\tau_{2}(0)=0$, we obtain the following solutions,

$$
\tau_{1}=\frac{1}{2 \mu \kappa^{2}}\left(1-e^{2 \mu y}\right), \quad \tau_{2}=\frac{1}{4 \mu^{3} \kappa^{2}}\left(\mu y-e^{2 \mu y}+\frac{1}{4} e^{4 \mu y}+\frac{3}{4}\right) .
$$

\footnotetext{
${ }^{5}$ When the brane position is taken at $y=y_{0}$, the warp factor is written as $A(y)=A\left(y_{0}\right) e^{-\mu\left|y-y_{0}\right|}$ and $A\left(y_{0}\right)=e^{-\mu y_{0}}$. And the brane runs from $y_{0}$ to the extended space direction $y>y_{0}$ according to the same flow equations as given here.
} 
Here we assumed $\tilde{\tau}_{2}=0$ for simplicity. In this case, we obtain

$$
\begin{gathered}
S_{b}^{\mathrm{eff}}=\int d^{4} x \sqrt{-\hat{g}}\left(\frac{1}{4 \mu \kappa^{2}} \hat{R}-\frac{1}{8 \mu^{3} \kappa^{2}}\left(-e^{2 \mu y}+\frac{1}{4} e^{4 \mu y}+\frac{3}{4}\right)\left(\hat{R}_{\mu \nu} \hat{R}^{\mu \nu}-\frac{1}{3} \hat{R}^{2}\right)+\cdots\right) \\
+S_{\mathrm{CFT}},
\end{gathered}
$$

where we used the following notations, $g_{\mu \nu}=A^{2}(y) \hat{g}_{\mu \nu}$ and $\hat{R}=R(\hat{g})$. This result implies that the gravitational constant is independent of $y$ and is equal to that given at $y=0$. Then the running given above does not alter the physics observed on the brane as far as the Einstein gravity is concerned.

As for the new higher derivative term or the Weyl term, the coefficient does not include the power series of $y$ like the anomaly term. Terms like $e^{2 \mu y}$ and $e^{4 \mu y}$ should be estimated by rewriting them as $e^{2 \mu\left(y-y_{0}\right)}$ and $e^{4 \mu\left(y-y_{0}\right)}$ near the boundary. They are not small, but the first Einstein term is in this case proportional to $A\left(y_{0}\right)^{2}=e^{-2 \mu y_{0}}$ which is large for $y_{0} \rightarrow-\infty$. Then, the higher derivative terms become negligible when $e^{2 \mu\left(y-y_{0}\right)} / A\left(y_{0}\right) \ll 1$ and $m^{2} / \mu^{2} \ll 1$. Under this condition, we can show the irrelevant terms are also negligibly small near the boundary. It would be possible to remove the constant $3 / 4$ by modifying the counter term $S_{\mathrm{CT}}$, but the situation depends on the renormalization condition of the effective action. In any case, we arrive at the following result,

$$
S_{b}^{\mathrm{eff}}=\int d^{4} x \sqrt{-\hat{g}}\left(\frac{A\left(y_{0}\right)^{2}}{4 \mu \kappa^{2}} \hat{R}+O\left(R^{2}\right)\right)+S_{\mathrm{CFT}},
$$

in the region of $y_{0}$ stated above. We call this action an invariant form under brane running since $\frac{A\left(y_{0}\right)^{2}}{4 \mu \kappa^{2}}$ is independent of the running brane position $y$. This implies that theories with different $\tilde{S}_{b}$ are considered to be the same when they are on the same flow line. An interesting example for such $\widetilde{S}_{b}$, which has the induced Einstein term in it, is the brane gravity model [25, 26, 27].

From (44), the $4 \mathrm{~d}$ Planck mass, $M_{\mathrm{pl}}^{2}=1 / 2 \kappa_{4}^{2}$, is related to the $5 \mathrm{~d}$ one, $M_{5}^{3}=1 / 2 \kappa^{2}$, as

$$
M_{\mathrm{pl}}^{2}=\frac{M_{5}^{3} A\left(y_{0}\right)^{2}}{2 \mu} .
$$

This condition implies a consequence that is of interest for phenomenological analysis: by assuming $M_{5} \approx \mu$, we find $M_{5} \ll M_{\text {pl }}$ since $1 \ll A\left(y_{0}\right)$. This might be an explanation of the hierarchy when the mass scale is given by $M_{5}$ for the field theory on the boundary.

In the next sub-section, we consider the case of positive $\lambda$ and find a similar invariant form in spite of the conformal non-invariance. However we give the discussion at $y_{0}=0$ for simplicity. 


\section{2 $\Lambda<0$ and $\lambda>0$}

Next is the case of $\Lambda<0$ and $\lambda>0$. Using $A(y)$ given in the previous section, the equation for $\tau_{1}$ is solved as

$$
\tau_{1}=\frac{\sinh \left(2 \mu\left(y_{H}-|y|\right)\right)+2 \mu y-b_{1}}{4 \mu \kappa^{2} \sinh ^{2}\left(\mu\left(y_{H}-|y|\right)\right)},
$$

where $b_{1}$ is an integration constant.

In this case, the conformal invariance is broken and we saw the flow of $\tau$ in section 3.2. However, $\tau$ of (21) is considered to be constructed by many $\tau_{i}$, and its $y$-dependence is determined by the infinite number of $\tau_{i}$. The same thing might be expected for $\tau_{1}$ given by (46). Actually we can see that (46) coincides with $\tau_{1}$ given in (25) in the limit of $\lambda=0$,

$$
\tau_{1} \rightarrow \frac{1}{2 \mu \kappa^{2}}
$$

as expected. But in general, $\tau_{1}$ in (46) deviates from the above value (47) for finite $\lambda$.

As in the previous subsection, we can estimate the effective brane action by adjusting $b_{1}$ such that $\tau_{1}(0)=0$ as in the case of $\lambda=0$. Then it is determined,

$$
b_{1}=\sinh \left(2 \mu y_{H}\right)
$$

As for $S_{\mathrm{CT}}$, for the $\lambda=0$ case, we can see that the first two terms in (41) are obtained by substituting the classical solution into the bulk action and integrating over $y$. Also for $\lambda>0$ case, we use this form of counter term, which cancels the UV divergent terms.

Replacing $S_{\mathrm{CT}}$ by the effective $4 \mathrm{~d}$ action obtained by substituting classical solution into the bulk action, we obtain

$$
S_{b}^{\mathrm{eff}}=\int d^{4} x \sqrt{-\hat{g}} \frac{1}{2 \kappa_{4}^{2}}\left(\hat{R}-6 \tilde{\lambda}+O\left(R^{2}\right)\right)+S_{\mathrm{QFT}}
$$

where

$$
\frac{1}{2 \kappa_{4}^{2}}=\frac{1}{2 \kappa^{2}} \int_{0}^{y_{H}} d y A^{2}(y), \quad \tilde{\lambda}=2 \lambda \kappa_{4}^{2} \int_{y}^{y_{H}} d y A^{2}(y) .
$$

The corresponding field theory is replaced by quantum field theory (QFT) since the bulk conformal symmetry would be broken. We notice here that the Planck constant $\kappa_{4}$ is exactly the same one with that given at $y=0$ [20]. While the effective cosmological constant $\tilde{\lambda}$ decreases with $y$ and it tends to zero at $y=y_{H}$. On the other hand, the geometry of the $4 \mathrm{~d}$ slice perpendicular to $y$ is charachterized by the scale factor, $a_{0}(t)=e^{\sqrt{\lambda} t}$. This implies that the decreasing of $\tilde{\lambda}$ is compensated by the higher derivative terms denoted by $O\left(R^{2}\right)$ in (49) [21, 22]. Then the equation (49) is well approximated by the first two terms in the region of $\tilde{\lambda} \sim \lambda$ or $y \sim y_{0}=0$.

This implies that the inflation is possible even if the cosmological constant is zero. In this case, however we should not neglect the higher derivative terms, which appear when 
the brane position is changed. Then, the higher derivative terms could be considered as a candidate of the dark energy, which sustains the inflation, instead of the cosmological constant. This point we will make more clear when discussing the case of two branes in the next section.

\section{Two parallel branes}

The system of two branes embedded in $\mathrm{AdS}_{5}$ bulk is considered in this section. The action is given as $S=S_{5}+S_{2 b}$, where

$$
S_{2 b}=-\tau_{\text {hid }} \int d^{4} x \sqrt{-g}-\tau_{v i s} \int d^{4} x \sqrt{-g} .
$$

The first (second) term represents the hidden (visible) brane with a plus (minus) tension $\tau_{\text {hid }}\left(\tau_{\text {vis }}\right)$ located at $y=y_{\text {hid }}\left(y=y_{v i s}<y_{H}\right)$. The bulk space between the two branes, $y_{\text {hid }} \leq y \leq y_{v i s}$, is projected on an orbifold $S^{1} / Z_{2}$; namely, the bulk action $S_{5}$ is defined by (II) in the compactified region.

The hidden brane is running from the original position $y=y_{\text {hid }}$ toward $y=y_{v i s}$ with the brane running method shown in the previous sections. The tension $\tau_{\text {hid }}(y)$ of the hidden brane at a running position $y$ is then obtained by (16) for $\lambda=0$ and (21) for $\lambda>0$. The visible brane, on the other hand, is fixed at $y=y_{\text {vis }}$ with the tension

$$
\begin{gathered}
\tau_{v i s}=-\frac{6 \mu}{\kappa^{2}} \text { for } \lambda=0, \\
\tau_{v i s}=-\frac{6 \mu}{\kappa^{2}} \sqrt{1+\frac{\lambda}{\mu^{2} A\left(y_{v i s}\right)^{2}}} \text { for } \lambda>0 .
\end{gathered}
$$

Let us consider the hidden brane at a running position $y$. In the effective braneworld action, $S_{2 b}^{\text {eff }} \equiv S_{2 b} / 2+S_{\mathrm{CT}}+S_{\mathrm{QFT}}$, $S_{\mathrm{CT}}$ is obtained with the classical approximation, i.e. by substituting the classical solution $A(y)$ into $S_{5}$, while quantum effects are included in $S_{\mathrm{QFT}}$. We then obtain the effective braneworld action for a running hidden brane:

$$
\begin{gathered}
S_{2 b}^{\mathrm{eff}}=\frac{1}{2} S_{2 b}+S_{5}^{\text {classical }}+S_{\mathrm{QFT}}, \\
S_{2 b}=-\int d^{4} x \sqrt{-g} \cdot \tau_{\text {hid }}(y)-\int d^{4} x \sqrt{-g} \cdot \tau_{v i s},
\end{gathered}
$$

where

$$
\tau_{\text {hid }}(y)=\tau_{0}+\tau_{1} R+\tau_{2}\left(R_{\mu \nu} R^{\mu \nu}-\frac{1}{3} R^{2}\right)+\tilde{\tau}_{2} R^{2}+\cdots,
$$

and in the limit $y \rightarrow y_{\text {hid }}, \tau_{i}(y)$ tends to $\tau_{\text {hid }}$ for $i=0$ and vanishes for other $i$. The explicit form of $S_{2 b}^{\mathrm{eff}}$ is obtained by calculating running parameters $\tau_{i}$ with the running brane method:

$$
S_{2 b}^{\mathrm{eff}}=\int d^{4} x \sqrt{-\hat{g}} \frac{1}{2 \kappa_{4}^{2}}\left(\hat{R}-6 \tilde{\lambda}+O\left(R^{2}\right)\right)+S_{\mathrm{QFT}}
$$


with

$$
\frac{1}{2 \kappa_{4}^{2}}=\frac{1}{2 \kappa^{2}} \int_{y_{h i d}}^{y_{v i s}} d y A^{2}(y), \quad \tilde{\lambda}=\lambda \frac{\kappa_{4}^{2}}{\kappa^{2}} \int_{y}^{y_{v i s}} d y A^{2}(y) .
$$

This effective action is obviously independent of $y$ in its first term. In general, on the other hand, the $\tilde{\lambda}$ term, the higher derivative terms and $S_{\mathrm{QFT}}$ depend on $y$. When the two branes are near the boundary, as already argued in the one-brane case, all the $y$ dependent terms are suppressed for small $m / \mu$. The effective action surely describes an inflation in $4 \mathrm{~d}$ spacetime with the net effect of $\tilde{\lambda}$ and the higher derivative terms, since the scale factor of the $4 \mathrm{~d}$ spacetime is $a_{0}(t)=e^{\sqrt{\lambda} t}$, independently of the position of the hidden brane.

In general, it is quite hard to calculate $S_{2 b}^{\text {eff }}$, since so is $S_{\mathrm{QFT}}$. This problem is circumvented by the following prescription. When the hidden brane is placed upon the visible one, i.e. at $y=y_{v i s}$, there is no bulk, so $S_{\mathrm{CT}}$ and $S_{\mathrm{QFT}}$ vanish. Then, $S_{2 b}^{\text {eff,ren }}$ is reduced to a calculable quantity, $S_{2 b} / 2$. Thus, we can evaluate $S_{2 b}^{\mathrm{eff}}$ by a hidden-brane running from the original position $y=y_{\text {hid }}$ to $y=y_{\text {vis }}$ []

The expectation value of $-S_{2 b}^{\mathrm{eff}}$ with the background field $a_{0}(t)$ can be regarded as the effective potential $V_{\text {eff }}$ for the brane positions, $y_{\text {hid }}$ and $y_{v i s}$. Now our discussion is focused on the case of small $\lambda$ and the brane running near the boundary. In this situation, the higher derivative terms, at least of order $\lambda^{2}$, are negligible compared with the leading term $R$ of order $\lambda$. For such small $\lambda$, the effective potential is given in the simple form

$$
V_{\text {eff }}\left(y_{\text {hid }}, y_{v i s}\right)=-a_{0}(t)^{3} \frac{6 \lambda}{\kappa_{4}^{2}} .
$$

The potential becomes small as the interbrane distance, $d=y_{v i s}-y_{\text {hid }}$, increases; note $\hat{R}=12 \lambda$ here and that this statement is valid for small $d$, since we consider the twobrane system near the boundary. This indicates that the two-brane system located near the boundary is unstable. This conclusion might be compatible with the radion stabilization analysis [23].

As for $\lambda=0, V_{\text {eff }}$ vanishes for any value of $d$, so $d$ is undetermined. The distance can be stabilized by introducing a massive bulk scalar [24]. The Goldberger-Wise stabilization mechanism is seen through the effective potential which has a minimum at a finite distance. As for a small and finite $\lambda$, however, (59) should be add to the Goldberger-Wise stabilization potential. There is a possibility that the net potential has no minimum, when (59) is larger than the stabilization potential in magnitude.

From a cosmological viewpoint, regarding the physics of the two-brane setting, let us finally point out how the two parallel branes are interconnected, in the case of a fixed separation distance. This is a consequence of Einstein's equations in the bulk, together with the junction conditions on the branes. Let us put $y_{\text {hid }}=0$. Then, with $\lambda_{0}=\kappa^{2} \tau_{0}^{2} / 36+\Lambda / 6$ and similarly $\lambda_{v i s}=\kappa^{2} \tau_{v i s}^{2} / 36+\Lambda / 6$ we obtain in the static case, 
taking the junction conditions

$$
\left.\frac{A^{\prime}}{A}\right|_{0}=-\frac{\kappa^{2}}{6} \tau_{0},\left.\quad \frac{A^{\prime}}{A}\right|_{v i s}=\frac{\kappa^{2}}{6} \tau_{v i s}
$$

into account, the following relationship [28]:

$$
\lambda_{v i s} A^{2}\left(y_{v i s}\right)=\lambda_{0}
$$

Since $A(y)<1$ when $y>0$ according to (18) (here assuming $A(0)=1$ ), we see that

$$
\lambda_{v i s}>\lambda_{0}
$$

which in turn means that $\tau_{v i s}^{2}>\tau_{0}^{2}$. As the effective four-dimensional cosmological constant $\lambda$ is equivalent to the presence of an isotropic fluid on the brane obeying the equation of state $p=-\rho$ for a 'vacuum' fluid [28], we see that the magnitude of the energy density is largest on the visible brane, $\left|\rho_{\text {vis }}\right|>\left|\rho_{0}\right|$. When the position of the hidden brane is running, the disturbance that this brane is subject to, has to influence the other brane also.

\section{Concluding remarks}

We have studied the effective braneworld action derived by brane running, for onebrane and two-brane models with and without a $4 \mathrm{~d}$ cosmological constant $\lambda$ on the brane. For the one brane model, the effective action is regularized such that it is finite at the boundary. In the limit $\lambda \rightarrow 0$, we can see the quadratic-curvature term, which can be regarded as the anomaly produced by the one-loop CFT (the AdS/CFT correspondence), in the action of the running brane. This term is however divergent at the boundary, so it must be cancelled by some regularization procedure. We give here such a regularization scheme. In the resultant effective braneworld action, the effective 4d Planck mass is invariant under the brane running for any value of $\lambda$. In the case of finite $\lambda$, interestingly, the $\lambda$ term diminishes and finally vanishes as the brane position inceases toward the horizon, while higher-derivative terms, and a cutoff QFT term, increase. This means that the inflation is realized by the higher-derivative and cutoff QFT terms even if $\lambda=0$, since the scale factor on the brane is always $a_{0}(t)=\exp (\sqrt{\lambda} t)$ during brane running.

When we consider the brane running near the boundary and the KK modes of small $m^{2}$ relative to $\mu^{2}$, the higher-derivative and cutoff QFT terms are suppressed, and the $\lambda$ term is almost constant. Thus, the brane running gives the same low-frequency physics at any brane position, as long as the conditions mentioned above are satisfied. The running brane action includes an "induced" gravity, since $\tau_{1}$ generally becomes finite as a result of brane running. Then it is not essential whether one takes into account the Einstein term in the brane action or not. 
It is hard to derive the definite form of $S_{\mathrm{QFT}}$ in the effective braneworld action. However, for the two brane model, we can get the effective braneworld action without $S_{\mathrm{QFT}}$, by moving the hidden-brane from the original position to the visible bane, since $S_{\mathrm{QFT}}$ finally vanishes due to the definition. Applying the action principle to the effective braneworld action thus calculated, we see that the two-brane system located near the boundary is unstable. This result might be compatible with the analysis of the radion stabilization [23. In the limit $\lambda \rightarrow 0$, the vacuum value of the effective braneworld action vanishes, so the separation between two branes is undetermined as expected from the restored conformal invariance.

We considered only the gravitational part, but we could extend the same analysis to the case where other fields are existing. Especially bulk scalars, which couple to the brane, will play an important role in the determination of distance between two branes in the RS I model. Another important problem to be studied is the estimate of massive

KK modes in the effective braneworld action. They are essential for the corrections of Newton's law and for some cosmological problems. We intend to discuss these points in a future paper.

\section{Acknowledgments}

This work has been supported in part by the Grants-in-Aid for Scientific Research (13135223, 14540271) of the Ministry of Education, Science, Sports, and Culture of Japan.

\section{References}

[1] J. Maldacena, Adv. Theor. Math. Phys. 2 (1998) 231, (hep-th/9711200).

[2] S.S. Gubser, I.R. Klebanov, and A.M. Polyakov Phys. Lett. B 428 (1998) 105, (hep-th/9802109).

[3] E. Witten, Adv. Theor. Math. Phys. 2 (1998) 253, (hep-th/9802150).

[4] A.M. Polyakov, Int. J. Mod. Phys. A14 (1999) 645, (hep-th/9809057).

[5] L. Randall and R. Sundrum, Phys. Rev. Lett. 83 (1999) 3370, (hep-ph/9905221).

[6] L. Randall and R. Sundrum, Phys. Rev. Lett. 83 (1999) 4690, (hep-th/9906064).

[7] A. Lewandowski, M. May and R. Sundrum, Phys. Rev. D67 (2003) 024036 (hep-th/0209050).

[8] A. Lewandowski, and M. Redi, (hep-th/0305013).

[9] M. Redi, JHEP 0305:032, 2003(hep-th/0304014). 
[10] N. Uekusa, (hep-th/0307107).

[11] M.J. Duff and J.T. Liu, Phys. Rev. Lett. 85 (2000) 2052, (hep-th/0003237).

[12] S. Nojiri,S. Odintsov and S. Zerbini, Phys. Rev. D62 (2000) 064006 (hep-th/0001192).

[13] W.D. Goldberger, and I.Z. Rothstein, Phys. Lett. B 491(2000)339, (hep-th/0007065).

[14] H. Georgi, A.K. Grant, G. Hailu, Phys.Lett. B506 (2001) 207-214, (hep-ph/0012379).

[15] K.A. Milton, S.D. Odintsov, S. Zerbini, Phys. Rev. D65 (2002) 065012 (hep-th/0110051).

[16] I. Brevik, K. Ghoroku, S. D. Odintsov and M. Yahiro, Phys. Rev. 66 (2002) 064016, (hep-th/0204066).

[17] S.B. Giddings, E. Katz and L. Randall, JHEP 03 (2000) 023, (hep-th/0009176).

[18] S.B. Giddings and E. Katz, (hep-th/0002091).

[19] M. Henningson and K. Skenderis, JHEP 07 (1998) 023, (hep-th/9806087).

[20] K. Ghoroku, and M. Yahiro, Phys. Rev. 66 (2002) 124020, (hep-th/0206128).

[21] A.A. Starobinsky, Phys. lett. 91B(1980), 99.

[22] A. Vilenkin, Phys. Rev. D32(1985), 2511.

[23] P. Binetruy, C. Deffayet and D. Langlois, Nucl. Phys. B615 (2001) 219.

[24] W.D. Goldberger, and M.B. Wise, Phys. Rev. Letters 83(1999), 4922.

[25] E. Kiritisis, N. Tetradis, T.N. Tomaras, (hep-th/0202037).

[26] G. Dvali, G. Gabadadze and M. Porrati, Phys. Letters B485 (2000), 208.

G. Dvali and G. Gabadadze, Phys. Rev. D63(2001), 065007.

[27] C. Csaki, J. Erlich, T.J. Hollowood and J. Terning, Phys. Rev. 63 (2001) 065019, (hep-th/0003076).

[28] I. Brevik, K. Børkje and J. P. Morten, gr-qc/0310103. 